\title{
New Pulsars Coincident with Unidentified Gamma-Ray Sources
}

\author{
Mallory Roberts, ${ }^{1}$ Scott Ransom, ${ }^{1}$ Jason Hessels, Margaret Livingstone, \\ Cindy Tam, Victoria Kaspi
}

Dept. of Physics, McGill University, Montréal, QC, H3A 2T8, Canada

\author{
Fronefield Crawford ${ }^{2}$ \\ Dept. of Physics, Haverford College, Haverford, PA 19041, USA
}

\begin{abstract}
We present the results of radio searches for pulsars within unidentified EGRET source error boxes. Using the Parkes multibeam system, we have surveyed 56 sources at Galactic latitudes $|b|>5^{\circ}$ which do not appear to be associated with blazars. This population has been suggested to be distributed like the local star forming region known as the Gould Belt, the Galactic Halo, and/or the millisecond pulsars. We have discovered several new pulsars in this survey, including three new binary systems.
\end{abstract}

\section{Introduction}

The EGRET mission has left us a legacy of unidentified $\gamma$-ray sources at low and mid-Galactic latitudes. Most of the $\sim 300$ known sources of emission above $100 \mathrm{MeV}$ remain unidentified, and most of these appear to be associated with the Galaxy (Hartman et al. 1999). Given that the only firmly established class of Galactic $\mathrm{GeV} \gamma$-ray sources is young $\left(\tau<10^{6} \mathrm{yr}\right)$ pulsars, it is natural to assume that many of the unidentified sources will turn out to be pulsars of some variety. All detections of $\gamma$-ray pulsations were first achieved by folding the data given an X-ray or radio ephemeris. While blind searches of GLAST data may result in some pulsar detections, the planned default scanning mode of observations will complicate such searches. Young pulsars tend to glitch and have significant timing noise, making coherent searches over several month long data sets problematic. For millisecond pulsars, especially if in a short period binary, there is little hope in blind searches of $\gamma$-ray data. Therefore, it is highly desirable to detect potential $\gamma$-ray pulsars before the next generation of highenergy $\gamma$-ray missions are launched.

The known $\gamma$-ray pulsars have hard $\gamma$-ray spectra. The unidentified sources that are bright above $1 \mathrm{GeV}$ tend to lie along the Galactic plane as would be expected for very young and energetic pulsars. Potential counterparts for many of

\footnotetext{
${ }^{2}$ also Department of Physics and Center for Space Research, MIT, Cambridge, MA 02139, USA

${ }^{2}$ with the Haverford College Pulsar Search Group, http://cs.haverford.edu/pulsar/
} 
the bright, hard, low-latitude sources have been identified, including young pulsars, pulsar wind nebulae, and maybe some microquasars (e.g., Roberts, Romani \& Kawai 2001). Unidentified sources that are faint above $1 \mathrm{GeV}$ have a large Galactic scale height (Lamb \& Macomb 1997). The unidentified sources at midlatitudes tend to have much larger error boxes (typically $\sim 1.5^{\circ}$ diameter $95 \%$ confidence contours) due to their softer spectrum and lower luminosity. This makes counterpart searching with X-ray imaging telescopes inefficient since it takes several pointings to cover an error box well. Archival X-ray and radio data are also fairly sparse compared to what is available at low-latitudes. Therefore, the proven strategy of identifying candidates through X-ray and radio imaging followed by deep pulsation searches is impractical for sources out of the plane.

\section{A Pulsar Survey of Mid-Galactic $\gamma$-ray Sources}

The spatial distribution of unidentified EGRET sources can be modeled as having a Gould Belt component plus a Galactic Halo component (Grenier 2002). Accordingly, there are at least two proposed classes of mid-latitude pulsars which might have detectable $\gamma$-ray emission. The Gould Belt is a local $(d \sim 50-300 \mathrm{pc})$ region of recent star formation in a rough disk inclined to the plane of the Galaxy where a significant number of young pulsars should have been born (Popov et al. 2003). The polar-cap $\gamma$-ray emission model allows for low-luminosity wide-angle emission coming from outside the primary beam with a softer spectrum than the direct polar cap emission (Harding \& Zhang 2001). Such "off-beam" $\gamma$-ray pulsars in the Gould Belt would have been detected by EGRET. Many would be radio-quiet, but a good fraction should be detectable as radio pulsars as well (Harding et al. 2004). A number of the Galactic Halo sources could be millisecond pulsars (MSPs). The distribution of MSPs outside of globular clusters is similar to that of the Galactic Halo unidentified EGRET sources, and some MSPs have spin-down energies and magnetospheric potentials in the range of the $\gamma$-ray emitting pulsars. There has also been a possible detection of a MSP by EGRET (Kuiper et al. 2000).

We have completed a survey of 56 unidentified EGRET sources for radio pulsations at $20 \mathrm{~cm}$ using the Parkes multibeam receiver. The 13 feeds of the receiver are placed two beam widths apart on the sky, allowing a four pointing tesselation pattern to have nearly complete coverage of an area of the sky $\sim 1.5^{\circ}$ across with partial coverage out to $\sim 2^{\circ}$, matching well the typical mid-latitude EGRET error box. The survey pointings were $\sim 35 \mathrm{~min}$ long, which is the same duration as the Parkes Multibeam (PMB) Galactic Survey (Manchester et al. 2001), and eight times that of the Swinburne mid- and high-latitude surveys (Edwards et al. 2001; Jacoby et al., these proceedings). We used a sampling time of $125 \mu \mathrm{s}$ to maintain sensitivity to MSPs, and performed full acceleration searches using the PRESTO search software (Ransom 2001) to have sensitivity to pulsars in even tight binary systems.

We used the following criteria to select our survey sources: $|l|>5^{\circ}$ (so as not to overlap with the PMB plane survey); no probable blazar counterpart as determined by Mattox, Hartman \& Reimer (2001); declination $<+20^{\circ}$ for easy accessibility from the Parkes telescope; and a $95 \%$ error contour radius as listed in the Third EGRET Catalog $\theta_{95} \lesssim 0.75^{\circ}$ so as to be well covered with a single 
tesselation pattern. Survey observations were performed between 2002 Jun and $2003 \mathrm{Jul}$, with confirmation observations still ongoing. Regular timing of newly discovered pulsars commenced in 2003 Jun. As of this writing, we have been able to solve the orbits for the newly discovered binary pulsars, but we do not yet have reliable $\dot{P}$ measurements for any of the new pulsars; hence, we are not yet certain if they are energetically capable of being $\gamma$-ray sources.

A total of 13 pulsars have been detected and confirmed so far in the survey. Six of the pulsars are new discoveries, and two pulsars in the survey area are listed in the ATNF catalog ${ }^{3}$ but were not redetected. Interestingly, only one pulsar (the 669-day binary PSR J0407+1607) was detected further than $\sim 50^{\circ}$ from the Galactic center (most of the pulsars are within $\sim 30^{\circ}$ ), despite more than half of the sources surveyed being outside this region. None of the pulsars are potential Gould Belt sources.

Only three new isolated pulsars were discovered, much fewer than were expected. The reason for this is probably man-made radio frequency interference (RFI). Although RFI masking was implemented and the Fourier spectra dereddened, it was obvious from the folding of candidates that, for long periods $(Z 200 \mathrm{~ms})$ and low trial dispersion measures $\left(\mathrm{DM} \lesssim 50 \mathrm{pc} \mathrm{cm}^{-3}\right)$, the $P-\dot{P}$ plane was filled with noise well above our sensitivity threshold. Unfortunately, this region of parameter space is exactly where we expect Gould Belt pulsars to lie. In order to test that the lack of new slow pulsar detections was not due to some unknown bias in the PRESTO software system, the data was searched independently for slow ( $>4 \mathrm{~ms}$ ) pulsars using the Sigproc/Seek (D. Lorimer, http://www.jb.man.ac.uk/ $\mathrm{drl} /$ ) software package. No new pulsars were discovered in the reanalysis. Additional reanalysis using much stricter RFI excision is planned.

\section{Binary Pulsars Discovered in EGRET Error Boxes}

The other three new pulsars appear to be recycled pulsars with low mass companions. A fourth binary pulsar, PSR J0407+1607, was detected which had previously been discovered at Arecibo (Lorimer et al., in preparation). This is the most productive survey yet for the number of binary pulsars detected per square degree of sky outside of globular clusters. A fifth binary pulsar is known within the survey area that was not redetected. Four of these five binary pulsars are within $30^{\circ}$ of the Galactic center, suggesting a deep survey of this region for binary pulsars would be very productive.

Remarkably, two of the new binary systems were discovered towards the same EGRET source, 3EG J1616-2221. PSR J1614-22 is a 3.15-ms pulsar in an 8.7-day orbit around a white dwarf companion. PSR J1614-23 is a 33.5-ms pulsar in a 3.15-day orbit with a minimum companion mass of only $0.08 M_{\odot}$. A 350-MHz observation of PSR J1614-23 with the GBT shows that the base of the pulse profile is very broad, perhaps suggesting a small inclination angle. The third new binary pulsar, PSR J1744-39, is at a Galactic latitude of $b=-5^{\circ} .3$, at the edge of the Parkes Multibeam Survey region, and was in fact independently

\footnotetext{
${ }^{3}$ See http://www.atnf.csiro.au/research/pulsar/psrcat/ .
} 
discovered in the recent reanalysis of the PMB survey data (A. Lyne, private communication). PSR J1744-39 is a 172 -ms pulsar in a 4.6 -hr binary system also with a minimum companion mass of $0.08 M_{\odot}$. This pulsar is notable for turning on and off on timescales of $\sim 100 \mathrm{~s}$, despite having a long period and a fairly high dispersion measure $\left(\mathrm{DM}=147.5 \mathrm{pc} \mathrm{cm}^{-3}\right)$. Continued timing of these sources will shortly determine whether they have the power to be the source of the observed $\gamma$-rays.

We have begun a northern extension using the GMRT at $610 \mathrm{MHz}$. The lower frequency should help maintain sensitivity to slower, nearby pulsars, allowing us to put meaningful limits on the number of Gould Belt pulsars.

Table 1. New pulsars discovered in our survey.

\begin{tabular}{lcccc}
\hline Pulsar & $\begin{array}{c}P \\
(\mathrm{~s})\end{array}$ & $\begin{array}{c}D^{a} \\
(\mathrm{kpc})\end{array}$ & $\begin{array}{c}P_{B}^{b} \\
(\text { days })\end{array}$ & $\begin{array}{c}M_{c}^{c} \\
\left(M_{\odot}^{c}\right)\end{array}$ \\
\hline $\mathrm{J} 1614-22$ & 0.003151 & 1.3 & 8.68 & 0.4 \\
$\mathrm{~J} 1614-23$ & 0.03350 & 1.9 & 3.15 & 0.08 \\
$\mathrm{~J} 1632-10$ & 0.7176 & $>50$ & $\ldots$ & $\ldots$ \\
$\mathrm{J} 1725-07$ & 0.2399 & 1.7 & $\ldots$ & $\ldots$ \\
$\mathrm{J} 1744-39$ & 0.1724 & 3.1 & 0.19 & 0.08 \\
$\mathrm{~J} 1800-01$ & 0.7832 & 1.6 & $\ldots$ & $\ldots$ \\
\hline
\end{tabular}

${ }^{a}$ Distance from the NE2001 dispersion measure model of Cordes \& Lazio (2002). ${ }^{b}$ Binary orbital period.

${ }^{c}$ Minimum companion mass.

\section{References}

Cordes, J. M., \& Lazio, T. J. W. 2002, astro-ph/0207156

Edwards, R. T. et al. 2001, MNRAS, 326, 358

Grenier, I. A. 2002, in Texas in Tuscany: XXI Symposium on Relativistic Astrophysics, eds. R. Bandiera et al., (Singapore: World Scientific), p. 397

Harding, A. K. \& Zhang, B. 2001, ApJ, 548, L37

Harding, A. K. et al. 2004, Adv. Sp. Res., 33, 571

Hartman, R. C. et al. 1999, ApJS, 123, 79

Kuiper, L., et al. 2000, A\&A, 359, 615

Lamb, R. C. \& Macomb, D. J. 1997, ApJ, 488, 872

Manchester, R. N. et al. 2001, MNRAS, 328, 17

Mattox, J. R., Hartman, R. C., \& Reimer, O. 2001, ApJS, 135, 155

Popov, S. B. et al. 2003, A\&A, 406, 111

Ransom, S.M. 2001, PhD thesis, Harvard University

Roberts, M. S. E., Romani, R. W., \& Kawai, N. 2001, ApJS, 133, 451 